Acta Crystallographica Section D

Biological

Crystallography

ISSN 0907-4449

\title{
Towards rationalization of crystallization screening for small- to medium-sized academic laboratories: the PACT/JCSG+ strategy
}

\begin{abstract}
A crystallization screening process is presented that was developed for a small academic laboratory. Its underlying concept is to combine sparse-matrix screening with systematic screening in a minimum number of crystallization conditions. The sparse-matrix screen is the cherry-picked combination of conditions from the Joint Center for Structural Genomics (JCSG) extended using conditions from other screens. Its aim is to maximize the coverage of crystallization parameter space with no redundancy. The systematic screen, a $\mathrm{pH}$-, anion- and cation-testing (PACT) screen, aims to decouple the components of each condition and to provide information about the protein, even in the absence of crystals, rather than cover a wide crystallization space. This screening strategy is combined with nanolitre-volume dispensing hardware and a small but practical experiment-tracking system. The screens have been tested both at the NKI and in other laboratories and it is concluded that they provide a useful minimal screening strategy.
\end{abstract}

\section{Introduction}

There has been a bifurcation within structural biology: one path is that of the high-budget structural genomics projects, which utilize specialized and capital-intensive equipment to meet the demands of their high-throughput laboratory pipelines; the other is that of the small academic laboratories, which use more traditional laboratory methods to produce structures. As the field of high-throughput structural biology has become more established, some of the techniques of the structural genomics initiatives have crossed over to academic laboratories. One of the easiest structural genomics lessons to implement is the adoption of standard protocols for various laboratory tasks. Crystallization would seem ideally suited to this approach, as there are no standard protocols. As we have little idea from the outset of a structure-determination program how to effectively tailor a crystallization strategy to a specific protein, any standard protocol would need to be effective for the 'general' protein.

The crystallization process can be broken down into screening and optimization steps: the desired result of screening is an indication of which further experiments need to be set up, while the desired outcome of an optimization experiment is reproducible well diffracting single crystals. For screening purposes, there are numerous commercial kits available: these tend to be collections of various solutions that have a proven track record in previous experiments (Jancarik \& Kim, 1991).

For any given set of solutions, there still are questions about what physical experiment should be set up, with vapour diffusion and microbatch being the two most popular types. If
Received 7 June 2005

Accepted 3 August 2005 
one decides on a vapour-diffusion experiment, then one must choose between sitting-, hanging- or sandwich-drop setups, each with a profusion of plastic plates and other options. It is important, especially in the context of automated highthroughput methodologies, to have established workflows. Various laboratories have done so (see, for example, Walter $e t$ al., 2005; Sulzenbacher et al., 2002) and we describe here a standard system put in place at the Netherlands Cancer Institute (NKI) that implements a practical procedure for crystallization screening suitable for small- to medium-sized academic laboratories. The basis of this system is a combination of commercially available technology and custom screens. The main aim is to maximize the chances of success whilst minimizing the cost of initial crystallization screening. This approach may be contrasted with the approach of setting up thousands of conditions from every available screen, which is expensive in terms of consumables, manpower and administration (recording what has been performed and monitoring the results). Our screening strategy does not aim to replace other screens, but instead provides an initial standard procedure that is tuned to both maximize the probability of success and to study the precipitation behaviour of the protein in a more systematic way. A distinct feature of this strategy, to our knowledge, is that it gives equal weight to sparse-matrix screening and systematic screening as an initial approach: the screens adopted in most laboratories that we are aware of mostly capitalize on the benefits of the crystallization space coverage of sparse-matrix approaches whilst ignoring or downplaying the potential benefits of a more systematic approach.

It has traditionally been difficult to judge the benefit of any given screen: a screen that enables the structure determination of a single recalcitrant protein might become favoured, although it may not be appropriate for other proteins. Now, however, the crystallization results from some of the largescale structural genomics projects are providing a basis for comparison of some of the commercially available screens by providing numbers of hits produced by each screen over the same large set of proteins (Page et al., 2003; Kimber et al.,

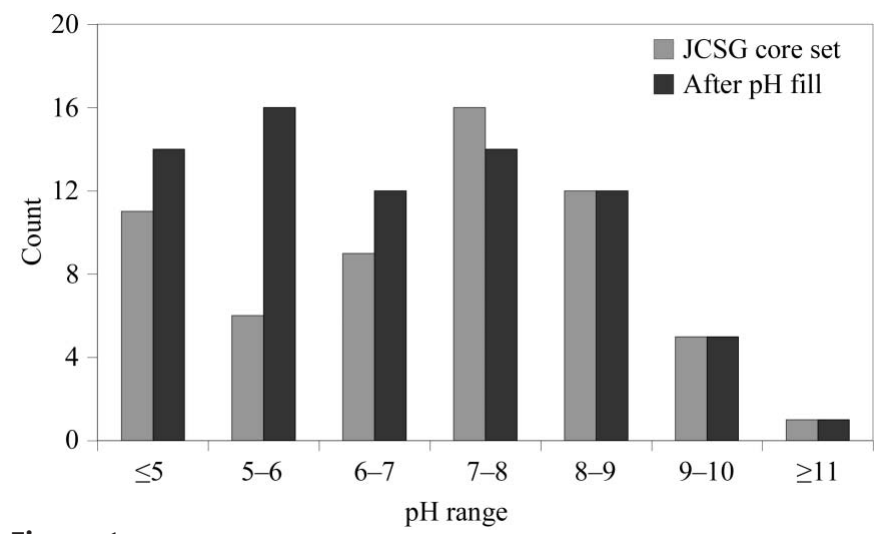

Figure 1

$\mathrm{pH}$ profiles of the JSCG core conditions and the final 96 solutions in the NKI sparse-matrix screen. The final screening set has a more extensive set of screening conditions around $\mathrm{pH}$ 6-7, where many proteins have been shown to crystallize in the past (Kantardjieff \& Rupp, 2004)
2003). In this study, we benchmark our strategy in a similar way but with a considerably smaller set of 'high-value' target proteins.

\section{Methods}

\subsection{Hardware and workflow}

It was determined from the outset that the robotic system put in place at the NKI should be robust enough to be easily used by all members of the laboratory, rather than requiring dedicated support. As a result of this, the machines chosen were quite specialized, as a machine dedicated to one task can be very simple to operate (flexibility in automation is generally achieved at the cost of complexity).

The screening solutions are stored in bar-coded 96 deepwell blocks. The blocks of screening solutions are either made up in-house on a standard liquid-handling machine (Tecan Miniprep 2) or purchased from a commercial vendor (Emerald BioSystems, Nextal). The crystallization setups are performed in CrystalQuick low-profile 96-well crystallization plates (Greiner Bio-one, catalogue No. 609171), as these provide a good optical platform for small drops. These plates are readily available and crystals can be harvested from the small drops in the shallow sample well relatively easily. The reservoirs of the crystallization plates are filled with $75 \mu \mathrm{l}$ crystallant solution in a single transfer from the deep-well source blocks using a 96-syringe Hydra (Matrix Technologies). Barcodes identify the individual crystallization plates and deep well blocks which, together with the macromolecular sample identification, are stored in a small custom-made database for experiment tracking. Protein and crystallant solutions (100 $\mathrm{nl}$ of each) are dispensed into each sample well using the Mosquito positive-displacement pipetting station (TTP Labtech) and the plates are sealed by hand with pressure-sensitive adhesive clear film (HR4-521, Hampton Research). The plates are loaded into an automatic inspection system (the BioStore from BIOTOM or the Crystal Farm from Bruker-AXS and Discovery Partners) and images of the drops are collected. If after a week no crystal hits have been found by the experimenter in these images, the plates are typically inspected by hand using an Olympus SZX12 stereomicroscope to ensure that nothing has been missed.

\subsection{Crystallization screens}

It was decided that the initial screening would be limited to two screens, each consisting of 96 different conditions, to minimize sample usage and cost. It was also decided that commercially available screens were not compatible with our philosophy for crystallization, owing to the high redundancy between different screen vendors coupled with lack of development of those screens based on crystallization statistics and the absence of a systematic screening protocol in a suitable format.

The first screen is a general sparse-matrix screen, in which the idea is to cover previously successful crystallization space (Jancarik \& Kim, 1991). The 96 conditions are based on the 
'core 67' results of the Joint Center for Structural Genomics (JCSG), in which a large number (473) of proteins from Thermotoga maritima were each set up against 480 conditions from 12 commercially available screens (Page et al., 2003). As two of the conditions identified in this 'core 67' are identical (Condition 1 from Crystal Screen and condition 1 from Crystal Screen Cryo, both from Hampton Research), 66 conditions were complemented with solutions from other commercially available screens, chosen to fill out the $\mathrm{pH}$ profile of the screen (Fig. 1) and to enhance the range of precipitants found in the screen. The 480 conditions used as the starting point in the JCSG study were all from well established screens and did not incorporate some of the newer crystallization chemicals such as sodium malonate (McPherson, 2001). We refer to our extended version of the JCSG core set as the JCSG+.

The second screen (PACT) is a systematic test of $\mathrm{pH}$, anions and cations with the precipitant polyethylene glycol (PEG). The rationale for the screen was the insight from investigators involved in high-throughput crystallization projects that the PEG/Ion screen from Hampton Research (HR2-126) gave more hits than any of the other commercial screens (Duncan McRee, personal communication; Walter et al., 2005). However, a caveat to the high hit rate is that many of the hits are redundant: a hit in the condition containing, say, ammonium formate is also likely to give a hit in a condition containing sodium formate, potassium formate or magnesium formate. Given this and given that the PEG/Ion screen does not contain buffers, a 96-well screen was designed which is essentially three screens in one: a 24-condition PEG/pH screen, a 24-condition cation/PEG screen and a 48-condition anion/PEG screen. The $\mathrm{PEG} / \mathrm{pH}$ portion of the screen uses multi-component buffer systems to produce a $\mathrm{pH}$ gradient from $\mathrm{pH} 4$ to $\mathrm{pH} 9$ within a given buffer system (Newman, 2004). Within this portion of the screen, the effect of $\mathrm{pH}$ change is decoupled from the presence of different ions in the crystallant solution, since all buffer ions are present in all tested pHs. This is repeated four times with different systems in order to break the ubiquitous $\mathrm{pH} / \mathrm{buffer}$ chemical correlation. The PEG/cation screen is less extensive than the PEG/ anion screen (six cations versus 12 anions tested) as cations generally affect the solubility of proteins less than anions (Carbonnaux et al., 1995). Within each mini-screen the type and concentration of the PEG is kept constant: $25 \%$ PEG 1500 in the PEG/pH screen, 20\% PEG 6000 in the PEG/cation screen and 20\% PEG 3350 in the PEG/anion screen. The choice was made to use different PEGs in each subscreen and to keep the concentration of the PEG within each subscreen constant. This is a compromise between the simplicity of using a single PEG at a single concentration and the complication of using the myriad of available PEGs at different concentrations.

The PACT screen was designed to not only reproduce the success in crystallization that was seen in the PEG/Ion screen from Hampton Research, but to allow the crystallographer to learn about the effects of different buffer/ion combinations on a protein in a systematic way. Information gleaned from this can be used to stabilize the protein for storage or to aid crystallization in other systems. A recent paper (Jancarik et al., 2004) describes an Optimal Solubility screening protocol in which a protein is tested against different buffer $/ \mathrm{pH}$ combinations in order to find a buffer in which the protein is soluble and monodisperse for crystallization experiments. After the first pass through buffer/pH testing, additives are included in the screening to maximize the monodispersity of the protein sample. The PACT screen results can be used in the same way: as there is a systematic testing of $\mathrm{pH}$ versus ions in the screen, the effect of cations, anions, $\mathrm{pH}$ and buffer chemistry on the solubility of the protein can be ascertained. The extensive use of different buffering systems in the PACT screen was deliberate so that buffer chemicals could be analyzed independently of $\mathrm{pH}$ and in combination with other buffering chemicals. There was no attempt to match the buffer with the salts in the PACT screen: the $\mathrm{pH}$ of the buffer is the $\mathrm{pH}$ of the buffer solution in isolation, not after mixing with the other components of the condition. Although every effort was made to keep the screen systematic, some solubility issues (with $\mathrm{Zn}^{2+}$ - and $\mathrm{PO}_{4}^{3-}$-containing conditions) forced some variation in ion concentration within the screen.

The sparse-matrix screen (JCSG+) and the PACT screen have been tested against proteins that we know crystallize at the NKI (http://xtal.nki.nl), as well as on a number of new projects that emerged in the NKI laboratory. After the results of the screens at the NKI were presented at a meeting (SPINE meeting, Munich, 2004), two other laboratories added the PACT screen into their screening protocols. At the Israel Structural Proteomics Center (ISPC; http://www.weizmann. ac.il/ISPC/) it is being used in microbatch crystallization experiments experiments with the Douglas Instruments Oryx-6 Crystallization robot together with the TriTek CrystalPro Visualization Robot and an integrated web-based database tracking system. At the Oxford Protein Production Facility (OPPF; http://www.oppf.ox.ac.uk) a variation of the PACT screen has been implemented in which the three different PEGs used as precipitants in the screen were replaced by $22.5 \%$ of a 'PEG smear'. The PEG smear is a mixture of ten polyethylene glycol polymers of various molecular weights ranging from 200 to 10000 (molecular weights of 200, 400, 600, 1000, 1500, 3000, 4000, 6000, 8000 and $10000)$. Equal volumes of $50 \%(w / v)$ solutions of the different PEGS were combined to give a stock solution that was $50 \%$ in PEG but has a molecular-weight range of 200-10 000. The PEG smear was formulated to investigate the possibility of rationalizing the myriad of PEGs available for crystallization experiments into a single stock for screening purposes. This simplifies making the screen, as well as removing the issue of which PEG to choose for initial screening. The concentration of $22.5 \%$ was chosen after analysis of the PEG concentrations found in the optimized conditions reported in the Protein Data Bank (http://www.pdb.org; Tom Peat, personal communication). If required, the smear could be deconvoluted into the component PEGs in subsequent optimization cycles. Results at the OPPF suggest that the results of PACT with the PEG smear are on a par with the PACT made with three single-molecular-weight PEGs initially implemented at the 
NKI. The OPPF have now, for convenience, adopted the commercial implementation of the standard PACT screen.

\section{Results}

\subsection{Hardware and workflow}

An in-house validation test of the Mosquito positivedisplacement pipetting station was run using the assay developed at the OPPF to test the performance of the dropdispensing machinery (Walter et al., 2003). Briefly, the test requires the nanodrop dispenser to dispense duplicate $100 \mathrm{nl}$ aliquots of fluorescein-doped liquids of varying viscosities. Both the precision and the accuracy of the machine are measured by comparing the fluorescence of the drops with a standard curve. A comparison of the accuracy of the Mosquito compared with the Oxford system is shown in Fig. 2(a) and details of the accuracy of dispense versus PEG 8000 concentration are shown in Fig. 2(b). The coefficient of variation (CV) for dispensing water was $4.7 \%$ and the average drop size dispensed was $99.7 \mathrm{nl}$ ( $c f .4 .2 \%$ and $98.9 \mathrm{nl}$, respectively, from the Oxford report). The Mosquito performed well with viscous solutions: the CV for $30 \%$ PEG 8000 was $6.2 \%$ and even for $40 \%$ PEG 8000 the CV was $17.8 \%$, but as shown in Fig. 2(b) there is a strong tendency to deliver inaccurate volumes in a manner that is dependent on the viscosity of the solution. A greater volume is delivered as the viscosity of the solution increases, possibly owing to carryover from the reservoir of high-viscosity liquids on the side of the tip. At the NKI the

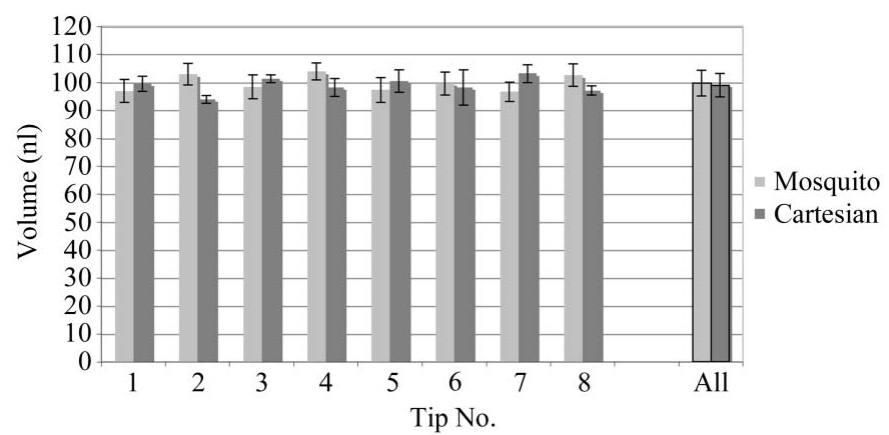

(a)

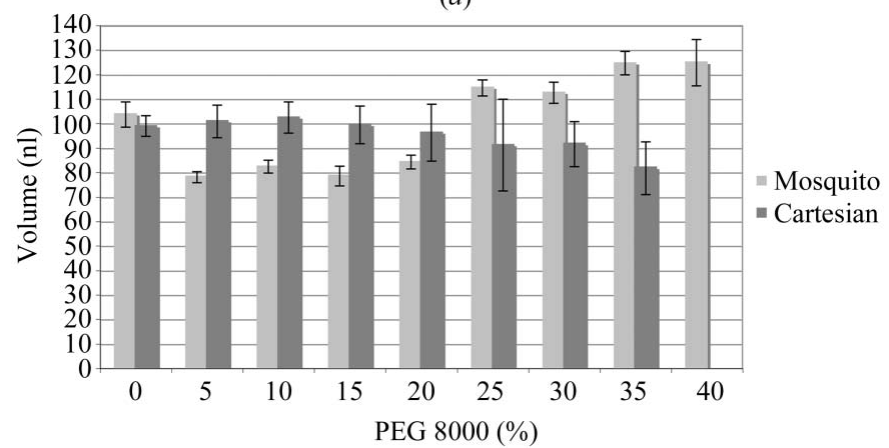

(b)

Figure 2

(a) Average volumes and standard deviations for pipetting $100 \mathrm{nl}$ water with the NKI and Oxford robotic systems. (b) Average volumes and standard deviations for pipetting increasing concentrations of PEG 8000 using the same systems. All experiments were repeated eight times. drops dispensed consist of $100 \mathrm{nl}$ protein which is combined with $100 \mathrm{nl}$ crystallant.

The barcode registration of the deep well and the crystallization plates is essential for experiment tracking through our custom-made database. The barcodes of the deep-well plates carry not only information about the chemicals in each block, but also provide the means of monitoring usage of the screens and hence aid timely ordering of replacement materials. A simple handheld barcode reader emulating keyboard input is used for barcode data entry. We are currently linking this database with the images database from the two imaging systems. It is foreseen that the custom-made experimenttracking database will be supplanted by the Protein Information Management System (PIMS) currently being developed in Europe (http://www.pims-lims.org).

\subsection{Crystallization screens}

Since the system was implemented, 34 new targets (proteins and protein complexes not crystallized before) from the NKI have been tried in the JCSG+/PACT screen combination. For all but two of these targets, other screens have also been tried (Table 1). In brief, 21 out of the 34 proteins produced crystals. Of these, 20 crystallized from the JSCG+/PACT combination alone, whilst only one needed extensive further screening to produce an initial crystal hit. It should be noted that not only the number but also the diversity of this test set is limited and the relatively high success rate is possibly obscured by the nature of the sample: 11 of the targets are AChBPs from different species and their complexes (all 11 crystallized), five are DNA-interacting proteins (four crystallized), two are protein kinases (none crystallized) and the remaining 16 are single proteins and complexes (11 different proteins in various covalent and non-covalent complexes make up these 16 targets) involved in the ubiquitination pathway (five crystallized).

In parallel with the evaluation of the screening strategy we present, ISPC and OPPF have tried the PACT screen as part of their in-house screening protocol (Walter et al., 2005). The JCSG+ was not implemented at these sites as their standard screening protocol includes many of the screens from which the JCSG+ conditions were selected. Of the 220 protein samples that were collectively tested between ISPC, OPPF and the NKI, 125 (57\%) crystallized (Table 2; Fig. 3). A significant proportion of these (57 of $220 ; 26 \%$ ) crystallized in the PACT screen. Eight of these proteins crystallized only in the PACT screen.

\section{Discussion}

\subsection{Hardware and workflow}

The Mosquito pipetting station from TTP Labtech was chosen as the drop-dispensing robot primarily because of its ease of use. In addition, the use of disposable positivedisplacement pipettes should enable this machine to cope with the variations in viscosity and surface tension that are found in the solutions used in crystallization. Although it could be 
Table 1

Statistics for the evaluation of the JCSG+ and PACT screening strategy.

Proteins tried

Proteins crystallized

Crystals in JCSG+/PACT

Crystals in JCSG+

Crystals in PACT

argued that the Mosquito is not as flexible as some other machines on the market, its dispensing speed and accuracy, in the context of the standardized setups at the NKI, compensate for this. It is easy to learn how to use the machine, owing mainly to its good user-interface design and implementation. The Mosquito has been robust following the initial period of installation and establishment of protocols. Overall, the Mosquito has proved to be a reliable instrument that performs on a par with other solutions, while having minimal maintenance requirements (owing to the absence of pumps, valves, system liquid etc.).

The Tecan liquid-handling station is most useful for reformatting screens (that is, transferring from $15 \mathrm{ml}$ tubes to deepwell blocks) and has also been used to make the PACT screen. The commercial availability of most screens in 96-well format at reasonable prices makes the investment for the liquidhandling robot unnecessary for crystallization, but its versatility and adaptability in a structural biology laboratory (protein-expression screening, DNA mini-preps, biochemical assays) should not be overlooked.

The Hydra is used to simplify the transfer of reservoir solutions from a deep-well block to an experimental plate (Walter et al., 2003). This machine is a well liked refinement to the crystallization protocol: however, a multi-channel pipette could be used for the transfer without sacrificing too much time or efficiency.

We strongly believe that the imaging system, although it might appear to be an unnecessary luxury to many laboratories, is essential for streamlining the whole process. Most importantly, images can be taken at regular time intervals. Imaging protocols can be standardized within the laboratory, without the need for extra working hours. Moreover, as we gain more experience and since the performance of the

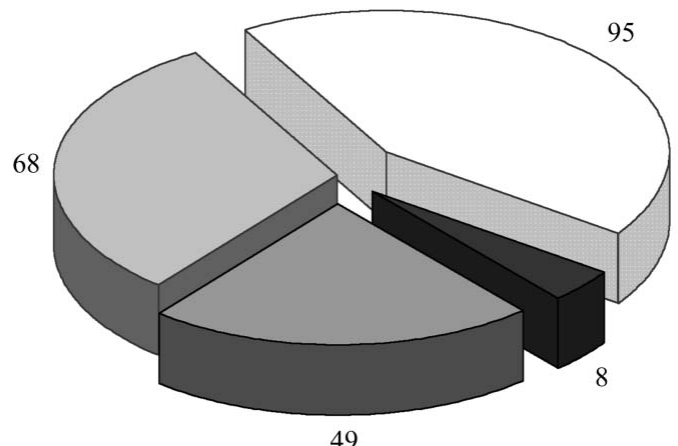

49

$\square$ Not crystallized $\square$ PACT only $\square$ PACT and non-PACT $\square$ Non-PACT only

Figure 3

A pie chart summarizing graphically the results from the PACT screen evaluation at NKI, OPPF and ISPC.
Table 2

Statistics for the evaluation of the PACT screen.

A graphical representation of these data is shown in Fig. 3.

\begin{tabular}{ll}
\hline Statistics per protein & \\
Proteins tried & 220 \\
Proteins crystallized (\% of total) & $125(57 \%)$ \\
Proteins not crystallized (\% of total) & $95(43 \%)$ \\
Proteins crystallized in non-PACT screens (\% of total) & $117(53 \%)$ \\
Proteins crystallized in PACT screen (\% of total) & $57(26 \%)$ \\
Proteins crystallized in non-PACT screen only (\% of total) & $68(31 \%)$ \\
Proteins crystallized in PACT screen only (\% of total) & $8(4 \%)$ \\
Statistics per drop & 284199 \\
Total drops tried & $125(0.044 \%)$ \\
Proteins crystallized (success rate; proteins/drop) & $1997(0.70 \%)$ \\
Drops with crystals (success rate; crystals/drop) & 34464 \\
PACT condition drops & $58(0.168 \%)$ \\
Proteins crystallized (success rate; proteins/drop) & $671(1.95 \%)$ \\
Drops with crystals (success rate; crystals/drop) & 249735 \\
Non-PACT conditions drops & $105(0.042 \%)$ \\
Proteins crystallized (success rate; proteins/drop) & $1326(0.53 \%)$ \\
Drops with crystals (success rate; crystals/drop) &
\end{tabular}

imagers has proved to be reliable over the last year, most users feel confident and trust the automatic imager, alleviating the need for direct plate inspection, even in the absence of crystals. Finally, the increase in the number of crystallization experiments that can be facilitated by the nanodrop robots will sooner or later lead to a need for the adoption of easier means of checking the experiments than manual inspection in the stereomicroscope: it is our belief that when setting up a high-throughput crystallization laboratory, the expense of the imaging system can be postponed but not alleviated.

\subsection{Crystallization screens}

The NKI test set comprises proteins from projects actively being pursued in that laboratory and includes various proteins and protein-protein complexes from the ubiquitin and SUMO signalling pathways, various species and complexes of the AChBP protein, eukaryotic proteins involved in retrotransposition, DNA-repair proteins and a couple of protein kinases. This sample is obviously biased (there are no extracellular proteins, for instance) and cannot be regarded as broad enough to draw statistically sound general conclusions, but it is indicative of the efficacy of the screens, especially in the context of laboratories that would be involved in similar projects. All (bar one) of the proteins that crystallized gave one or more crystals in the minimal PACT/JCSG+ screening set. The protein that did not crystallize in the initial screening set required 576 additional conditions until small star-shaped crystals were identified. Even after extensive optimization these crystals did not diffract beyond $4.0 \AA$ and the structure is yet to be solved.

The success rate of the screening strategy we present was sufficient for it to be adopted as the primary screen for all NKI projects. Should no crystals be obtained, other screens are tried. Which screens are tried subsequently is determined by the judgment of the researcher associated with the project.

The PACT screen has been evaluated more extensively through the SPINE collaboration. 220 protein samples were collected which are relevant to the general thematic areas of 
the SPINE proteomics initiative: human pathogens (bacterial and viral proteins), cancer-related targets and targets relevant to neurological development and neurodegenerative disease. Of these 220 'high-value' targets that were collectively tested between ISPC, OPPF and the NKI, 125 (57\%) crystallized. A significant proportion of these $(57 ; 26 \%)$ crystallized in the PACT screen. Eight of these proteins crystallized only in the PACT screen. Although this is only $4 \%$ of the total number, it demonstrates that PACT not only samples 'conventional' crystallization space but also has the potential to explore less charted regions. It should be noted that the PACT experiments were only $12 \%$ of the total experiments (34 464 out of 281031 ), yet gave crystals in $26 \%$ of the targets; the success rate of the PACT screen was 1.7 proteins crystallized per 1000 conditions tried compared with 0.4 proteins crystallized per 1000 conditions for the other screens. The PACT screen alone has therefore about four times the success rate of the average screen across these three sites, although there is some bias in favour of the PACT screen as the 'other screens' were not as systematically tested against all the proteins. The first set of screens always included at least two (for 26 targets), three (for 34 targets) and in most cases more than five (for 160 targets) screens other than PACT. Thus, in the cases in which the protein did not crystallize immediately more conditions from 'other screens' were tried, thus lowering the success rate of the other screens. If we consider the total number of crystals produced by each of the screens (rather than the number of crystallized proteins as above), 671 of the 1997 crystals (33\%) were from the PACT screen and it also gave about four times more crystals per 1000 experiments compared with other screens. Unfortunately, this statistic is also biased by the internal redundancy of the PACT screen; if crystals are obtained at $\mathrm{pH} \mathrm{7,} \mathrm{crystallization} \mathrm{is} \mathrm{also} \mathrm{likely} \mathrm{at} \mathrm{pH} 8$ under identical conditions. Despite these biases, the statistics suggest that the PACT screen is useful not only for complementing but also extending currently available crystallization screening space. Furthermore, for proteins that do not produce crystal hits in the PACT screen, the rational design of the screen still allows the extraction of stabilization and solubility information as described by Jancarik et al. (2004).

The automation effort, combined with the screening strategy presented here, has made a dramatic difference in the NKI. As in many laboratories, the relative speed and ease of setting up crystallization experiments using robotics initially resulted in a considerable increase in consumables required and a proportionate rise in cost. Although less protein is used per experiment (approximately $12 \mu \mathrm{l}$ per 96 conditions) and each single experiment (condition) is cheaper in the nanovolume 96-well experiment than the traditional microvolume 24-well plate, there is a tendency to compensate by setting up many more experiments in the expectation that this will increase the chance of success. In response to this the strategy developed at the NKI is to minimize the initial screening to a limited set of conditions that not only widely cover crystallization parameter space but also attempt to describe the behaviour of the protein. This achieves a significant reduction in operating costs, whilst not adversely affecting the ultimate success rate of proteins that are refractory to this initial screening strategy; these can then be tested afterwards against all the standard screens in the usual way.

\section{Availability}

The PACT screen as described in this publication is available from Molecular Dimensions Ltd as 'PACT premier'. The JCSG+ screen and the PACT screen as described in this publication are available from Nextal Biotechnologies as the 'JCSG+ suite' and 'PACT suite', respectively. Details of the PACT and JCSG+ screens are available from the vendors' websites, the SPINE website (http://www.spineurope.org) and as supplementary material ${ }^{\mathbf{1}}$. The in-house database for deep well and crystallization tray tracking is available on request from the corresponding author.

This work was funded in part by the European Commission as SPINE contract No. QLG2-CT-2002-00988 under the Integrated programme 'Quality of Life and Management of Living Resources'. The instrumentation at the NKI was funded by a generous internal grant to AP and Professor Titia K. Sixma. MBJ is funded by the European Commission BIOXHIT project under the 6th Framework Programme thematic area 'Life Sciences, Genomics and Biotechnology for Health', contract No. LHSG-CT-2003-503420. DIS and the OPPF are funded by the UK MRC. IB is funded by the eHTPX initiative of the UK BBSRC. The ISPC is funded by the Israel Ministry of Science and Technology and the Divadol Foundation. JLS is the incumbent of the Morton and Gladys Pickman Professorship of Structural Biology.

\section{References}

Carbonnaux, C., Riès-Kautt, M. \& Ducruix, A. (1995). Protein Sci. 4, 2123-2128.

Jancarik, J. \& Kim, S.-H. (1991). J. Appl. Cryst. 24, 409-411.

Jancarik, J., Pufan, R., Hong, C., Kim, S.-H. \& Kim, R. (2004). Acta Cryst. D60, 1670-1673.

Kantardjieff, K. A. \& Rupp, B. (2004). Bioinformatics, 20, 2162-2168.

Kimber, M. S., Vallee, F., Houston, S., Necakov, A., Skarina, T., Evdokimova, E., Beasley, S., Christendat, D., Savchenko, A., Arrowsmith, C. H., Vedadi, M., Gerstein, M. \& Edwards, A. M. (2003). Proteins, 51, 562-568.

McPherson, A. (2001). Protein Sci. 10, 418-422.

Newman, J. (2004). Acta Cryst. D60, 610-612.

Page, R., Grzechnik, S. K., Canaves, J. M., Spraggon, G., Kreusch, A., Kuhn, P., Stevens, R. C. \& Lesley, S. A. (2003). Acta Cryst. D59, 1028-1037.

Sulzenbacher, G. et al. (2002). Acta Cryst. D58, 2109-2115.

Walter, T. S., Diprose, J., Brown, J., Pickford, M., Owens, R. J., Stuart, D. I. \& Harlos, K. (2003). J. Appl. Cryst. 36, 308-314.

Walter, T. S., Diprose, J. M., Mayo, C. J., Siebold, C., Pickford, M. G., Carter, L., Sutton, G. C., Berrow, N. S., Brown, J., Berry, I. M., Stewart-Jones, G. B. E., Grimes, J. M., Stammers, D. K., Esnouf, R. M., Jones, E. Y., Owens, R. J., Stuart, D. I. \& Harlos, K. (2005). Acta Cryst. D61, 651-657.

\footnotetext{
${ }^{1}$ Supplementary material has been deposited in the IUCr electronic archive (Reference: CY5025). Details for accessing these data are given at the back of the journal.
} 